\title{
PRESCRIBING SPECTACLES
}

BY

\author{
A. S. Percival
}

NEWCASTLE-UPON-TYNE

From time to time there is an outcry by ophthalmologisis against the prescribing optician, but it must be noted that the opticians generaliy have raised their standard, and those in the first rank know more about optics than we ophthalmologists as a class do. It is only when we have shown by our work that we are better at prescribing spectacles than the best optician that we can regain our leading position at refraction work.

It is not the estimation of the refractive error that is so frequently at fault, but attention to little details in the prescription that I wish to speak about now.

A fortnight ago a patient complained of her reading glasses, she had taken them back to the well-known ophthalmologist who prescribed them, but after examination he pronounced them to be all right, and said that she must learn to use them. I fcund that the refractive error and the presbyopia were quite accurately corrected $(\mathrm{R}+2 \mathrm{D}, \mathrm{L}+5 \mathrm{D})$, each glass, moreover, was normally centred, and these centres were opposite her pupils in the primary position. But when reading she depressed her eyes $14^{\circ}$ of $15^{\circ}$, and consequently her fixation lines traversed her lenses about $\tau \mathrm{mm}$. below their centres. (For the centre of rotation of the eye is situated about $27 \mathrm{~mm}$. behind the first focal plane of the eye, the situation of the spectacles, and $2 \tau \tan 14 \frac{1}{2}{ }^{\circ}=7 \mathrm{~mm}$.) Hence the line of print she was reading was depressed $\frac{\mathrm{Dl}}{10}$ or $\frac{2 \times 7}{10}$ or $1.4 \nabla$ for her right eye and $\frac{5 \times 7}{10}$ or $3.5 \nabla$ for her left eye. In other words when reading she suffered from a right hyperphoria of $2.1 \nabla$ induced by her spectacles. Fither the glasses should be lowered by raising the bridge $7 \mathrm{~mm}$. so that the fixation lines should pass through the optical centres of each glass, or better the left glass should be decentred down $4 \mathrm{~mm}$. according to the well-known formula $1=\frac{10 \mathrm{~N}}{\mathrm{D}}$ or $\frac{21}{5}$. It would be foolish to order a decentration of $4.2 \mathrm{~mm}$. as this would entail much needless labour and expense. This alteration in her left glass relieved her of all her previous trcuble. But what I want to insist upon is this, that the ophthalmologist should have noted this point about the decentration on his prescription; it is not good for his reputation, which he has deservedly won as a most accomplished and successful operator. 
It will be found that attention must be paid to decentration in all reading spectacles for anisometropes. But do not correct a heterophoria that does not grive rise to any symptoms; in fact only order a correction if it is found on trial to give relief. Enough about decentration; I dealt thoroughly with the subject in 1921 iBrit. Jl. Ophthal. Vol. V., pp. 453-9).

Some thoughtful attention should be devoted to the subject of periscopic effect. For instance, should $+3 \mathrm{D}$ sph. $-6 \mathrm{D}$ cyl. ax. $180^{\circ}$ or $-3 \mathrm{D}$ sph. $+6 \mathrm{D}$ cyl. ax. $90^{\circ}$ be prescribed? In the first case when the cylinder is placed next the eye it must be remembered that the plane axis is horizontal, and as the periscopic effect is most desired in the horizontal direction, the second form with the concave spherical next the eye is far the better. Had the axis of the cylinder been against the rule say $+3 \mathrm{D}$ sph. $-6 \mathrm{D}$ cyl. ax. $90^{\circ}$, this form would be the best with the cylindrical surface facing the eye; for now a concave surface of $-6 \mathrm{D}$ in the horizontal direction would be next the eye.

Periscopic lenses are fairly easy to order if there is no astigmatism, but when astigmatism is present toric surfaces must be ordered, and as toric surfaces are only ground on a $3 \mathrm{D}, 6 \mathrm{D}$ or a 9D base (positive or negative) much ingenuity will be required in getting the best possible result.

For large convex menisci $41 \mathrm{~mm}$. in width and $1.2 \mathrm{~mm}$. thick at the edge $(\mu=1.523)$, it is well to remember that very satisfactory periscopic lenses can be made for close work with the concave surface of $-5.5 \mathrm{D}$ facing the eye between the powers of $+4 \mathrm{D}$ and $+8 \mathrm{D}$. Indeed, within these limits the eye can range throughout a solid angle of $60^{\circ}$, i.e. $30^{\circ}$ in any direction from the middle line and have perfect sight, the radius of the circle of confusion being less than that of a macular cone.

For accurate work one must remember that with a convex meniscus the second principal point is in front of the lens, so that the back focal distance (B.F.D.), measured from the axial point on the concave surface in the anterior focal plane of the eye, is rather less than the actual focal length of the lens. For instance, a periscopic lens of axial thickness $4.5 \mathrm{~mm}$. with anterior surface $+12.5 \mathrm{D}$, posterior surface $-5.5 \mathrm{D}$ will have the effect of a +7.5D lens (not a +7D lens), for its B.F.D. is $-133.7 \mathrm{~mm}$. As the concave surface next the eye and so $f_{2}{ }^{\prime \prime}$ are known, and $t$ the thickness of the meniscus for a given strength is also known, one puts B.F.D. equal to the second focal distance of the lens required, and the value of the convex surface can be found from the formula

$$
\text { B.F.D. }=\frac{f_{2}^{\prime \prime}\left(t+f_{i}^{\prime \prime}\right)}{t+f_{1}^{\prime \prime}-f_{2}^{\prime}}
$$

These glasses will not be periscopic for distance; I find that between the range of $+6 \mathrm{I})$ and $+9 \mathrm{D}$ the ocular, concavity must be that of $\mathrm{a}-7 \mathrm{D}$ lens. 
As an example, suppose a periscopic lens of $+6 \mathrm{D}$ be required for distance, the power of the ocular curvature will be $-7 \mathrm{D}$ if $\mu=1.523$, and $t=3.8 \mathrm{~mm}$., what will be the power of the anterior surface?

$$
\mathrm{BFD}=\frac{f_{2}^{\prime \prime}\left(t+f_{1}\right)}{t+f_{1}^{\prime \prime}-f_{2}^{\prime}}=\frac{f_{2}^{\prime \prime} x}{x+\mu f_{2}^{\prime \prime}}
$$

When $x=t+f_{1}^{\prime \prime}$, and $f_{1}^{\prime \prime}$ must be found

$$
\text { We have } \frac{1}{\mathrm{BFD}}=\frac{1}{f_{2^{\prime \prime}}}+\frac{\mu}{x}
$$

or if $\mathrm{D}$ represents the power required $(+6.5)$ and if $d$ denote the power of the ocular curvature $(-7)-.001(\mathrm{D})=-.001(d)+\frac{\mu}{x}$

$$
\text { or } x=\frac{-\mu}{.001(\mathrm{D}-d)}
$$

This is the general formula; in this case $x=\frac{-1.523}{.001(6+7)}=\frac{-1523}{13}=-117.154=3.8+f_{1}{ }^{\prime \prime} \cdot \therefore f_{1}^{\prime \prime}=$ -120.954 and the power of the anterior surface should be $\frac{-1000 \mu}{f_{1^{\prime \prime}}}$ $=\frac{1523}{120.954}=+12.59 \mathrm{D}$.

From an examination of the much advertised Punctal lenses I find that the refractive error as well as the astigmatic error is far greater in the peripheral part of the field than in my pattern.

In conclusion I may briefly give the method of converting a sphero-cylinder into toric form, as it is rarely given in the books. I should point out that the method makes no correction for the position of the second principal point, so the result is not mathematically accurate.

I'rescribing Toric Lenses. Write the sphero-cylinder as two crossed cylinders. Thus if the sphero-cylinder be $+3.5 \mathrm{D}$ sph. $+3.5 \mathrm{D}$ cyl. ax. $90^{\circ}$ write it as $+3.5 \mathrm{D}$ cyl. ax. $180^{\circ}$ and $+7 \mathrm{D}$ cyl. ax. $90^{\circ}$. Call the lower cylinder L, and the higher cylinder $\mathrm{H}$.

\section{(I) Compound Astigmatism}

(1) When the base is of the same sign as that of the cylinders Subtract the value $L$ of the lower cylinder from the base and denote the result by $\mathrm{A}$, or $\mathrm{A}=\mathrm{B}-\mathrm{L}$.

Add $A+L$ and the result is one cylinder, in fact the base,

Add $\mathrm{A}$ to $\mathrm{H}$ and the result is the other cylinder for the toric surface.

The spherical surface is given by - A.

For instance, suppose the given sphero-cylinder for reading be +3.5 sph. $+3.5 \mathrm{D}$ cyl. ax. $90^{\circ}$ or $+3.5 \mathrm{D}$ cyl. ax. $180^{\circ}$ and $+7 \mathrm{D}$ cyl. ax. $90^{\circ}$. 
It will then be found that the best result will be obtained on a $+9 \mathrm{D}$ base.

Then $\mathrm{A}=\mathrm{B}-\mathrm{L}=9-3.5=5.5$.

$\mathrm{A}+\mathrm{L}=5.5+3.5=+9 \mathrm{D}$ cyl. ax. $180^{\circ}$.

$\mathrm{A}+\mathrm{H}=5.5+7=+12.5 \mathrm{D}$ cyl. ax. $90^{\circ}$.

'The prescription is written thus :--

$$
\frac{-5.5 \mathrm{D} \text { sph. }}{+9 \mathrm{D} \text { cyl. ax. } 180^{\circ} \text { and }+12.5 \mathrm{D} \text { cyl. ax. } 90^{\circ}}
$$

As noted above this will give an excellent periscopic result if the glass is only required for close work.

(2) When the base is of opposite sign to that of the cylinders let $\mathrm{A}=\mathrm{B}-\mathrm{H}$, and then proceed as before.

Suppose that we wish to write the same sphero-cylinder in toric form on a $-6 \mathrm{D}$ base.

$\mathrm{A}=\mathrm{B}-\mathrm{H}=-6-\mathrm{7}=-13$.

$\mathrm{A}+\mathrm{H}=-13+7=-6 \mathrm{D}$ cyl. ax. $90^{\circ}$.

$\mathrm{A}+\mathrm{L}=-13+3.5=-9.5 \mathrm{D}$ cyl. ax. $180^{\circ}$.

and we obtain $\frac{+13 \mathrm{D} \text { sph. }}{-6 \mathrm{I}) \text { cyl. ax. } 90^{\circ} \text { and }-9.5 \mathrm{D} \text { cyl. ax. } 180^{\circ}}$

This would be a better periscopic form than the first one for distance.

\section{(II) Mixed Astigmatism}

Take the difference between the base and the value of that cylinder which is of opposite sign and call it A.

Suppose that we write $-1 \mathrm{D} \mathrm{sph} .+3 \mathrm{D}$ cyl. ax. $90^{\circ}$, or -11 cyl. ax. $180^{\circ}$ and $+2 \mathrm{D}$ cyl. ax. $90^{\circ}$, in toric form on a $+3 \mathrm{D}$ base

Then $\mathrm{A}=3-(-1)=4$.

$4-1=+3 \mathrm{D}$ cyl. ax. $180^{\circ}$, and $4+2=46 \mathrm{D}$ cyl. ax. $90^{\circ}$ and the prescription is

$$
\frac{-4 \mathrm{D} \text { sph. }}{+3 \mathrm{D} \text { cyl. ax. } 180^{\circ} \text { and } \div 6 \mathrm{D} \text { cyl. ax. } 90^{\circ}}
$$

This would be the best periscopic form for this glass with the present available toric lenses, but there would be a very fair periscopic range even without using torics at all, i.e. with the - 1D curvature facing the eye and the $+3 \mathrm{D}$ cylinder anterior.

What I protest about is the not uncommon practice of the ophthalmologist baldly giving the sphero-cylinder which corrects the error of refrartion and then adding "give periscopic form." It is only comparable to a doctor writing instead of a prescription to a chemist "give something for rheumatism."

Finally, there is a warning I should add; some few cases cannot wear periscopic lenses, they complain of the surface that they look at appearing curved: I have not yet got to the root of this trouble. Most patients who have once worn periscopic lenses will never be content with the simple correction again. However, for lenses of low power there is no necessity to order the neriscopic form. 